\title{
Performance Evaluation of Xpert MTB/RIF Assay and Prevalence of rpoB Gene Mutation in Mycobacterium Tuberculosis in Clinical Isolates Quetta, Pakistan: A Cross Sectional Retrospective Cohort Study
}

\author{
Ashraf Ullah Khan ${ }^{1}$, Shereen Khan ${ }^{2}$, Hidayatullah ${ }^{3}$, Zabi Ullah ${ }^{4}$ \\ ${ }^{1}$ Faculty of Pharmacy and Health Sciences, University of Balochistan (Main Branch) \\ Quetta, Pakistan. \\ Email: ashrafstanikzai [AT] gmail.com \\ ${ }^{2}$ Department of Pulmonology, Fatima Jinnah General and Chest Hospital, \\ Quetta, Pakistan \\ Email: shereenqta [AT] yahoo.com \\ ${ }^{2}$ Department of Pulmonology, Fatima Jinnah General and Chest Hospital, \\ Quetta, Pakistan \\ Email: drhidayatullah2011 [AT] yahoo.com \\ ${ }^{4}$ Faculty of Curative Medicine, Malalay Institute of Higher Education \\ Aino Maina, Kandahar, Afghanistan \\ Email: zabiullahkhanstanikzai2015 [AT] yahoo.com
}

\begin{abstract}
With the use of Xpert assay, the detection mechanism of tuberculosis has completely transformed. Concurrently detecting mycobacterium and its resistance to rifampin (rif) which is also one of the primary marker for MDR-TB in addition to its surrogate activity against MTB in first line drugs. At large, resistance of mycobacterium strains against rifampicin is caused by rpoB gene mutations about $96.1 \%$ globally while these mutations are generally situated at a site of 507-533 rd residuals of amino acid in the rpoB gene of Mycobacterium, this region is also termed as Rifampicin resistance determining region (RRDR). In this study, we determined the mutations of various rpoB genes in different probes of 81 bp of RRD Region. A total of 6353 different specimens obtained at provincial reference laboratory of Fatima Jinnah general and chest hospital, Quetta (FJHQ), between Jan 2016 and Apr 2018 were examined by Xpert MTB/RIF assay. Altogether positive and negative samples for MTB were further examined with the light emitting diode (LED) fluorescence microscope. Pearson Chi square test was used to find out the associations between probe type and gender, treatment history and age group of the patients. Out of 6353 specimens 1297 MTB positive cases were detected by Xpert assay including 184 samples having both mycobacterium and RIF-resistance advised by mutations in 81 bp-RRD Region of rpoB gene which is further divided into five different probes. Further LED fluorescence microscope detected and confirmed 327 positive MTB samples out of 1297 specimens of Xpert assay. The associated probes mutation for rifampicin resistance were as follow: $E(98 / 184), D(23 / 184), B(15 / 184), C(5 / 184)$, $A$ (3/184), two probe mutation (5/184) in $D \& E$ (3/184) and $C \& D$ (2/184) and all probe mutation (35/184). The percentage of rpoB gene mutation in the studied population was $14.187 \%$. The commonest mutation associated with rpoB gene was in Probe $E$ (53.2608\%) also termed as 531 and 533 codons, additionally two probe mutation (2.7173\%) in $D \& E(1.6304 \%)$ and $C \& D(1.0869 \%)$ and all probe mutation $(19.0217 \%)$ were also detected that has never been detected before globally.
\end{abstract}

Keywords- RRDR, rpoB, 81 bp, Xpert® MTB/RIF

\section{INTRODUCTION}

With the use of Xpert assay, the detection mechanism of tuberculosis has completely transformed, concurrently detecting mycobacterium and its resistance to rifampin [1-2], which is also one of the primary marker for MDR-TB [3] in addition to its surrogate activity against tuberculosis in first line drugs against tuberculosis. The rifampicin inhibits the synthesis of DNA dependent RNA by binding with beta $(\beta)$ subunit of RNA polymerase enzyme as a result Mycobacterial protein production is blocked. At large, resistance of mycobacterium strains against rifampicin is caused by rpoB gene 
mutations about $96.1 \%$ globally while these mutations are generally situated at a site of 507-533 ${ }^{\text {rd }}$ residuals of amino acid in the rpoB gene of Mycobacterium, this region is also termed as Rifampicin resistance determining region (RRDR) [4]. In this study, we identified the frequency of RIF-resistant MTB in Quetta using Xpert assay and further determined the mutations of various rpoB genes in different probes of $81 \mathrm{bp}$ of RRD Region.

There are about 22 high ranked TB burden countries globally and Pakistan counts $5^{\text {th }}$ among them. The frequency of MDR TB in new cases and previously treated MDR-TB patient was $4 \%$ and $35 \%$ respectively according to WHO report [5]. Our result varies greatly with WHO's statistics as we have find out an increase number of MDR-TB in cases 105/184 $(57.1 \%)$ as compared to the previously treated MDR-TB patient $79 / 184(42.9 \%)$, this might be due to the variance in biological features and concerned drug resistance according to different geographical sites [6-7]. There is limitation of data prevalence on mutation in rpoB gene in Quetta, so this study is aimed to offer a reference line data on various mutations in different rpoB genes of RRD Region using Xpert assay. For the most accurate diagnosis of rifampicin resistance in various mycobacterium strains the finding of mutation in various rpoB gene and RRD region is very important [8]. In this study we primarily aimed to investigate the occurrence of rifampicin resistant mycobacterium strains as well as to investigate the mutations in rifampicin resistant determining region (RRDR) of RNA polymerase $\beta$ subunit (rpoB) gene in MTB by Xpert assay.

\section{MATERIALS AND METHODS}

We have studied overall 6353 samples conducting this study. Each sample was received and analyzed as per standard WHO protocol.

\section{ETHICAL ISSUES}

The concerned research project was approved and permitted by the authorities of FJHQ and incharge microbiology laboratory. The focal person and head of pulmonology department also collaboratively agreed to this study.

\section{CLINICAL SPECIMENS}

The total number of specimens included in the study was 6353. These specimens were obtained from suspected patients with MTB infections and were referred to microbiology (BSL-2) laboratory, FJHQ for routine diagnostics between Jan 2016 and Apr 2018. This laboratory is the solitary TB culture and Xpert assay laboratory situated in FJHQ, a provincial referral laboratory serving urban, peri-urban and rural population of about 12.34 million people around the Provence. All the referring samples had protocols received at microbiology laboratory approved by the authorities of Fatima Jinnah general and chest hospital, Quetta.

After receiving these specimens at microbiology laboratory, all the important details of patient were noted and specific characteristics of specimen (including consistency and volume) were logged into the data receiving form of concerned reference microbiology laboratory, the concerned data that was present in hard-form was transferred into computerized database soft-form, the computer room was access restricted area. The samples received at provincial reference laboratory included sputa, nasopharyngeal aspirates, gastric aspirates, pleural fluid and cerebrospinal fluid. The first choice for the diagnosis of tuberculosis at provincial reference laboratory of FJHQ was Xpert assay and LED fluorescence microscopy. LED fluorescence microscopy was done as confirmatory test along with Xpert assay.

The collected specimen was prepared for Xpert ${ }^{\circledR}$ MTB/RIF assay as per WHO protocol First the specimen was taken in falcon tube and isopropanol and sodium hydroxide $(\mathrm{NaOH})$ solutions concentrated $(10 \%-20 \%)$ and $(5 \%-10 \%)$ respectively was added to the sample at $(1: 2)$ ratio, after that the sample is vortexed and then centrifuged at 1000 RPM for five minutes. In the next step 2-3 ml sample taken from the supernatant is added to cartridge and loaded in Xpert assay and the result is noted after 2 hours. Concentrated Smears is also prepared and stained from the sample according to the standard WHO protocol with Auramine-O-phenol and analyzed by an LED fluorescence microscope under 200X and 400X magnification. The leftover specimen was stowed at freezer needed in case of occurrence of any error in test performance or other requested diagnostic tests. The suspicious samples were further stained with Ziehl- Neelsen (ZN) staining for confirmation. Figure 1 shows Frequency of probes mutation in rpoB gene detected by the Xpert assay. 


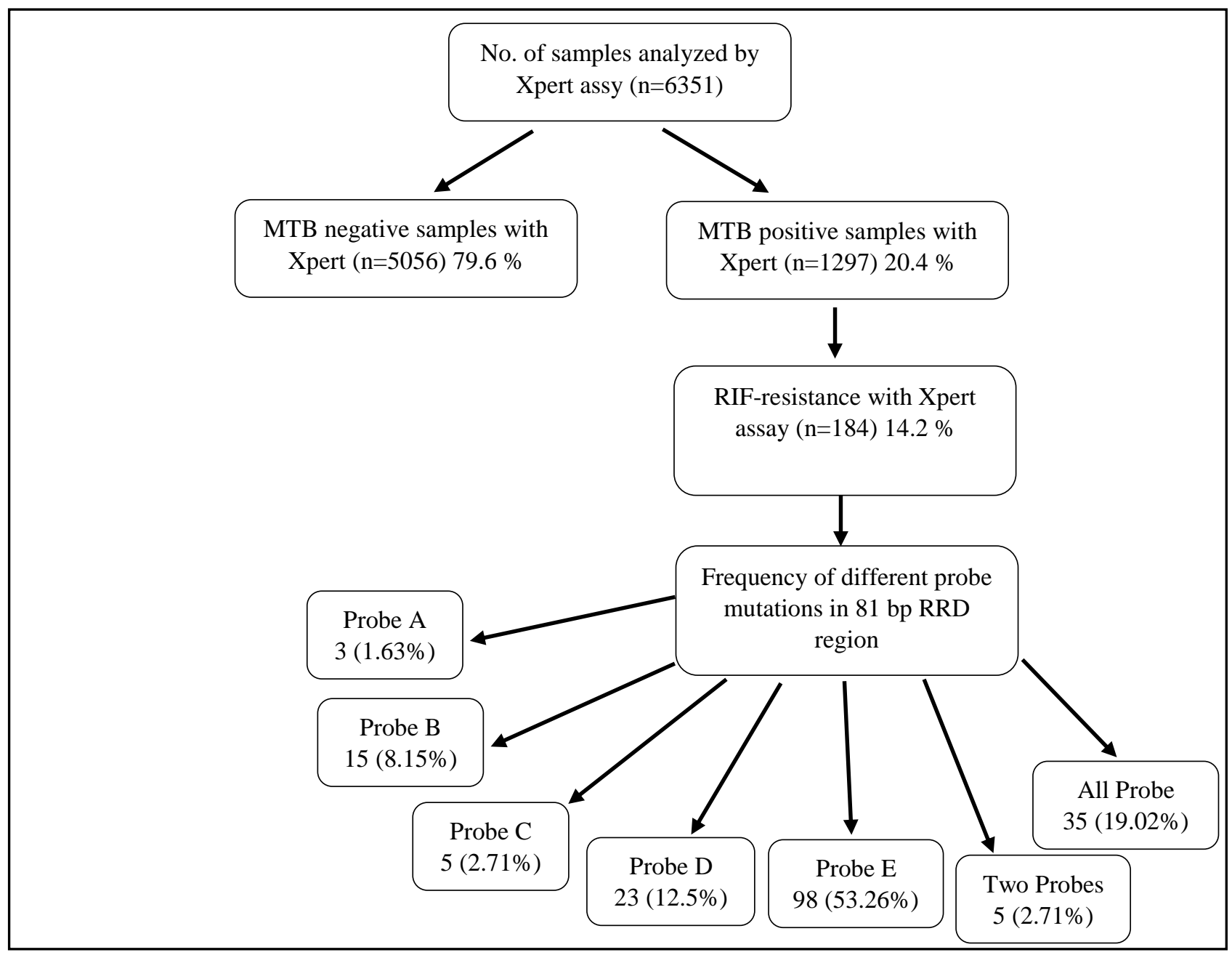

Figure 1: Frequency of probes mutation

\section{XPERT® MTB/RIF ASSAY}

All the specimens were analyzed by Xpert MTB/RIF assay G4 version 5 and the results were compared with LED fluorescence microscope. Xpert assay was further used to detect rifampicin resistant MTB. The comparison between Xpert assay and LED fluorescence microscope gave us reference for the reliability of Xpert assay over LED fluorescence microscope.

\section{LED FLUORESCENCE MICROSCOPY}

The LED fluorescence microscopy was done for all the received sample as per standard manufacturer instructions and WHO protocol.

\section{STATISTICAL ANALYSIS}

The inferential statistics were applied on the collected data using IBM SPSS version 23. Statistically significant associations were found between probe type and gender and treatment history, while non-significant between probe type and age group of patients using Pearson Chi square test.

\section{RESULTS}

184 specimens were detected by Xpert ${ }^{\circ}$ MTB/RIF assay having rifampicin resistant MTB. The resistance of rifampicin was advised by mutation in five dissimilar probes present in 81 base pairs (bp) rifampicin resistance determining region (RRDR) of rpoB gene of mycobacterium genome. The mutation in these five different probes were detected by Xpert assay denoted by capital alphabets A, B, C, D, and E. The RIF-resistance frequency associated with probes were as follow: E (98/184), D (23/184), B (15/184), C (5/184), A (3/184), two probe mutation (5/184) in D\&E (3/184) and C\&D (2/184) and all probe mutation $(35 / 184)$.

There was similarity of about $25.212 \%$ for detection of MTB by Xpert® MTB/RIF assay and LED fluorescence microscope while Xpert ${ }^{\circledR}$ MTB/RIF is much more reliable than LED fluorescence microscope. RIF-resistance detection 
by Xpert assay along with its detection of MTB provides a key path for the treatment. The average contamination rates for Xpert assay throughout the tenure was 3\%. Out of 1297 MTB positive samples, 970 patients were not detected by LED fluorescence microscopy. There was no sample lost to contamination. We also report that Xpert@ MTB/ RIF assay had generally better sensitivity than microscopy and is more reliable and trust worthy than concentrated smear microscopy [9]. Table 1 shows Frequency of probes for various rpoB gene mutations in the $81 \mathrm{bp}$ RRDR of rpoB gene in rifampicin resistance detected (RRD) cases of Mycobacterium tuberculosis with different demographic representation.

Table 1: Frequency of probes for various rpoB gene mutations in the 81 bp RRDR of rpoB gene in rifampicin resistance detected (RRD) cases of Mycobacterium tuberculosis with different demographic representation.

\begin{tabular}{|c|c|c|c|c|c|c|c|c|c|}
\hline \multirow[t]{2}{*}{ Demography } & \multicolumn{8}{|c|}{ Probe types } & \multirow[t]{2}{*}{ Total (\%) } \\
\hline & $\mathrm{A}$ & B & $\mathrm{C}$ & $\mathrm{D}$ & $\mathrm{E}$ & $\mathrm{D} \& \mathrm{E}$ & $C \& D$ & All & \\
\hline Frequency $(\%)$ & $3(1.6)$ & $15(8.1)$ & $5(2.7)$ & $23(12.5)$ & $98(53.4)$ & $3(1.6)$ & $2(1.0)$ & $35(19.0)$ & $184(100)$ \\
\hline \multicolumn{10}{|l|}{ Gender } \\
\hline Female & 2 & 9 & 3 & 17 & 69 & 0 & 0 & 16 & $116(63)$ \\
\hline Male & 1 & 6 & 2 & 6 & 29 & 3 & 2 & 19 & $68(37)$ \\
\hline \multicolumn{10}{|l|}{ History } \\
\hline New patients & 1 & 7 & 4 & 8 & 72 & 2 & 2 & 9 & $105(57)$ \\
\hline Previously treated & 2 & 8 & 1 & 15 & 26 & 1 & 0 & 26 & $79(43)$ \\
\hline \multicolumn{10}{|c|}{ Age categories (Year) } \\
\hline$<10$ & 0 & 1 & 0 & 1 & 2 & 0 & 0 & 1 & $5(2.7)$ \\
\hline $10-20$ & 1 & 3 & 2 & 5 & 9 & 1 & 0 & 2 & $23(12.5)$ \\
\hline $21-30$ & 0 & 2 & 1 & 7 & 23 & 0 & 0 & 12 & $45(24.4)$ \\
\hline $31-40$ & 0 & 1 & 0 & 4 & 9 & 0 & 0 & 4 & $18(10)$ \\
\hline $41-50$ & 1 & 5 & 1 & 5 & 18 & 1 & 1 & 9 & $41(22)$ \\
\hline $51-60$ & 1 & 2 & 1 & 0 & 19 & 1 & 1 & 5 & $30(16)$ \\
\hline $60-70$ & 0 & 1 & 0 & 1 & 15 & 0 & 0 & 1 & $18(9.7)$ \\
\hline$>70$ & 0 & 0 & 0 & 0 & 3 & 0 & 0 & 1 & $4(2)$ \\
\hline
\end{tabular}

\section{DISCUSSION}

The studies conducted worldwide concluded that the genetic basis of drug resistance against MTB is a specific type of point mutation in some of the most significant genes like katG, rpoB, embB and rpsL [4]. MDR-TB is one of the biggest threat to human worldwide particularly to individuals living in industrialized or technologically advanced countries. We used Xpert assay in our study to detect the RIF-resistance in MTB due to the mutation in rpoB gene. Previous studies showed that RIF-resistance is accompanied by isoniazid resistance in 90\% cases of MDR-TB [10-11]. Balochistan is the biggest province of Pakistan with over 12.34 million population, it also shares the major portion of its border with Afghanistan and Iran. This study is significant due to the use of Xpert assay for the prevalence of rpoB gene mutation in mycobacterium. According to our information a retrospective study like this has never been done before in this region. This study is the first of its kind conducted in Quetta. This study will provide the initial reference data in the event if the range of RIF-resistant TB was stretched to the other cities of Balochistan.

The frequency of mutation in rpoB gene in 81 bp RRD Region was as follow: the most common was in codons 531 (53.2608\%), $526(12.5 \%), 513(8.1521 \%), 522(2.7173 \%)$ and $511(1.6304 \%)$. These were termed as probes E, D, B, C 
and A according to the Xpert assay, we have also reported two probe mutation $(2.7173 \%)$ in 626 \& 531 codons (i.e. D \& $\mathrm{E}=1.6304 \%$ ) and $522 \& 526$ codons (i.e. $\mathrm{C} \& \mathrm{D}=1.0869 \%)$ and all probes mutation $(19.0217 \%)$. A similar study was conducted by Yue et al. (2003) in china and reported these frequencies as follow: $531(41 \%), 526(40 \%)$, and $513(4 \%)$ [11]. A similar study by Ullah et al. (2016) conducted at Khyber Pakhtunkhwa (Pakistan) reported as follow: 531 (77\%), $513(10.8 \%), 526(8.3 \%), 511(1.2 \%)$, and 522 (1.5\%) [12]. A report from Uganda by Mboowa et al. (2014) also suggested that the most common mutation found at RRD Region of MTB at Kampala was in codon 531 (58\%) [13]. A study by Khan et al. (2013) conducted at Punjab, Pakistan reported mutations in various probes of RRDR in MTB as follow E (52\%), B $(15 \%)$ and $\mathrm{D}(7.0 \%)$ [14]. After analyzing and comparing all the previously conducted studies we come to a conclusion that the codon 531 also known as probe $\mathrm{E}$ is the most predominant probe associated with rifampicin resistance. According to the Boehme et al. (2011) and Moure et al. (2011) the sensitivity and specificity of mycobacterium for identification of RIF-resistance was 94.4-100\% and 98.3-100\% [15-16].

In this study, we found out that mycobacterium was $14.1865 \%$ resistant to rifampicin in clinical isolates of Quetta. Our findings were related to Liu et al. (2013) as they reported the frequency of mycobacterium resistance to at least one of the first line drugs is 30.4\% [17]. The conclusion of Luiz et al. (2013) regarding mycobacterium resistance to at least one of the first line drugs in clinical isolates of Brazil was $44.3 \%$, these conclusions are higher than our findings [18]. Javaid et al. (2008) and Ullah et al. (2016) pointed the mycobacterium resistance as $11.3 \%$ and $11.5 \%$ to at least one of the first line drugs in clinical isolates of various regions of Pakistan. These statistical findings are less than our conclusion in Quetta which means that MDR cases in Quetta, are more than any other province of Pakistan [19-20]. This might be due to the favorable windy weather of this region which facilitates the air born disease like Tuberculosis. The prevalence of multi drug resistant Tuberculosis according to the survey done by the WHO in Pakistan to minimize the threat and maximize the safety against MDR TB reported $4.3 \%$ in new case while $19.4 \%$ in previously treated cases [5]. The WHO's report also varies greatly with our findings, this variance might be as a result of geographical location or favorable atmosphere for the air born disease.

The program for efficient detecting of MTB in clinical isolates was extended by WHO in 2012. This extension was done in countries with maximum TB patients and Xpert assay was preferred for effective diagnosis of MTB than the carbol fuchsine $\mathrm{ZN}$ staining and auramine phenol fluorescence microscopy [5]. Hence the worthwhile instrument for detection, isolation and effective treatment of tuberculosis is Xpert ${ }^{\circledR}$ MTB/RIF assay particularly in regions with maximum MDR TB cases such as Quetta.

\section{CONCLUSIONS}

The percentage of rpoB gene mutation in the studied population was $14.187 \%$. The commonest mutation associated with rpoB gene was in Probe E (53.2608\%) also termed as 531 and 533 codons, additionally two probes mutation in D \& E $(1.6304 \%)$ and C \& D (1.0869\%) and all probes mutation (19.0217\%) were also reported that has never been reported before globally. For efficient and potent therapy against MDR-TB additional molecular studies in Quetta must be done involving various other molecular techniques to find out unknown facts about drug resistance and its increased frequency in Quetta. The unique feature of Xpert assay is that it is much more sensitive than LED fluorescence microscopy, can detect RIF-resistance and is easy to operate. Xpert MTB/RIF assay will always remain irreplaceable tool due to its crucial platform of RIF-resistance detection.

\section{ACKNOWLEDGMENT}

We acknowledge Medical Superintendent and all the staff of Pulmonology department FJHQ, from the core of our heart for their factual guidance, facilitation, support and collaboration while conducting this study.

\section{AUTHORS' CONTRIBUTION}

The absolute manuscript was read and approved by all the authors. The experiments was Conceived, designed and performed by: Ashraf Ullah. Analysis of the data and paper writing was done by: Zabi Ullah. Technical support, collaboration and supervision was provided by: Shereen Khan and Hidayatullah.

\section{CONFLICT OF INTEREST}

The authors declare that there is no conflict of interests regarding the publication of this article.

\section{ABBREVIATION}

FJH, fatima jinnah general and chest hospital; MTB, mycobacterium tuberculosis; MDR-TB, multi drug resistant tuberculosis; RIF, rifampicin/rifampin; RRD-Region, rifampicin resistance determining region; rpoB , RNA Polymerase Beta subunit; RRD cases, rifampicin resistance detected cases.

\section{FUNDING}

We have received no fund for this study. 


\section{AVAILABILITY OF DATA AND MATERIALS}

The manuscript contains complete data supporting our findings and results.

\section{REFERENCES}

1. Helb D, Jones M, Story E, Boehme C, Wallace E, Ho K, Kop J, Owens MR, Rodgers R, Banada P, Safi H, Blakemore R, Lan NT, Jones-López EC, Levi M, Burday M, Ayakaka I, Mugerwa RD, McMillan B, Winn-Deen E, Christel L, Dailey P, Perkins MD, Persing DH, Alland D: Rapid detection of Mycobacterium tuberculosis and rifampin resistance by use of on-demand, near-patient technology. J Clin Microbiol 2010, 48:229-37.

2. Boehme CC, Nabeta P, Hillemann D, Nicol MP, Shenai S, Krapp F, Allen J, Tahirli R, Blakemore R, Rustomjee R, Milovic A, Jones M, O'Brien SM, Persing DH, Ruesch- Gerdes S, Gotuzzo E, Rodriques C, Alland D, Perkins MD: Rapid molecular detection of tuberculosis and rifampin resistance. N Engl J Med 2010, 363:1005-15.

3. Drobniewski FA, Wilson SM: The rapid diagnosis of isoniazid and rifampin resistance in Mycobacterium tuberculosis: a molecular story. J. Med. Microbiol 1998, 47:189-96.

4. Ramaswamy S, Musser JM: Molecular genetic basis of antimicrobial agent resistance in Mycobacterium tuberculosis. Tuberc Lung Dis 1998, 79:3-29.

5. Organization WH. World Health Organization Global Tuberculosis Report 2013. Geneva, Switzerland: World Health Organization, WHO Press; 2013.

6. Li W, Wang S, Li C, Liu Y, Shen G, Zhang X, Niu T, Gao Q, Van Soolingen D, Kremer K. Molecular epidemiology of Mycobacterium tuberculosis in China: a nationwide random survey in 2000. Int J Tuberc Lung Dis. 2005;9(12):1314-9.

7. Brossier F, Veziris N, Truffot-Pernot C, Jarlier V, Sougakoff W. Performance of the genotype MTBDR line probe assay for detection of resistance to rifampin and isoniazid in strains of Mycobacterium tuberculosis with lowand highlevel resistance. J Clin Microbiol. 2006;44(10):3659-64.

8. Ocheretina O, Escuyer VE, Mabou M-M, Royal-Mardi G, Collins S, Vilbrun SC, Pape JW, Fitzgerald DW. Correlation between genotypic and phenotypic testing for resistance to rifampin in Mycobacterium tuberculosis clinical isolates in Haiti: investigation of cases with discrepant susceptibility results. PLoS One. 2014;9(3):e90569.

9. Jones-López E, Manabe YC, Palaci M, Kayiza C, Armstrong D, Nakiyingi L, Ssengooba W, Gaeddert M, Kubiak R, Almeida JP, Alland D, Dietze R, Joloba LM, Ellner JJ, Dorman SE: Prospective cross-sectional evaluation of the small membrane filtration method for diagnosis of pulmonary tuberculosis. J Clin Microbiol 2014, 52(7):2513-20.

10. Ioerger TR, Koo S, No E-G, Chen X, Larsen MH, Jacobs Jr WR, Pillay M, Sturm AW, Sacchettini JC. Genome analysis of multi-and extensively-drug-resistant tuberculosis from KwaZulu-Natal, South Africa. PloS One. 2009;4(11):e7778.

11. Yue J, Shi W, Xie J, Li Y, Zeng E: Mutations in the rpoB Gene of MultidrugResistant Mycobacterium tuberculosis Isolates from China. J Clin Microbiol 2003, 41(5):2209-12.

12. Ullah I, Shah AA, Basit A, Ali M, Khan A, Ullah U, Ihtesham M, Mehreen S, Mughal A, Javaid A. Rifampicin resistance mutations in the 81 bp RRDR of rpoB gene in Mycobacterium tuberculosis clinical isolates using Xpert MTB/RIF in Khyber Pakhtunkhwa, Pakistan: a retrospective study. BMC Infect Dis. 2016; 16:413.

13. Mboowa G, Namaganda C, Ssengooba W. Rifampicin resistance mutations in the 81 bp RRDR of rpoB gene in Mycobacterium tuberculosis clinical isolates using Xpert MTB/RIF in Kampala, Uganda: a retrospective study. BMC Infect Dis. 2014; 14(1):481.

14. Khan SN, Niemann S, Gulfraz M, Qayyum M, Siddiqi S, Mirza ZS, Tahsin S, Ebrahimi-Rad M, Khanum A. Molecular characterization of multidrug resistant isolates of Mycobacterium tuberculosis from patients in Punjab, Pakistan. Pakistan J Zool. 2013;45(1):93-100.

15. Boehme CC, Nicol MP, Nabeta P, Michael JS, Gotuzzo E, Tahirli R, Gler MT, Blakemore R, Worodria W, Gray C. Feasibility, diagnostic accuracy, and effectiveness of decentralised use of the Xpert MTB/RIF test for diagnosis of tuberculosis and multidrug resistance: a multicentre implementation study. The lancet. 2011;377(9776):1495-505.

16. Moure R, Muñoz L, Torres M, Santin M, Martín R, Alcaide F. Rapid detection of Mycobacterium tuberculosis complex and rifampin resistance in smearnegative clinical samples by use of an integrated real-time PCR method. $J$ Clin Microbiol. 2011;49(3):1137-9.

17. Liu Q, Zhu L, Shao Y, Song H, Li G, Zhou Y, Shi J, Zhong C, Chen C, Lu W. Rates and risk factors for drug resistance tuberculosis in Northeastern China. BMC Public Health. 2013;13(1):1171.

18. Luiz RSS, Suffys P, Barroso EC, Kerr LRFS, Duarte CR, Freitas MVC, Mota RMS, Frota CC. Genotyping and drug resistance patterns of Mycobacterium tuberculosis strains observed in a tuberculosis high-burden municipality in Northeast, Brazil. Braz J Infect Dis. 2013;17(3):338-45.

19. Javaid A, Hasan R, Zafar A, Ghafoor A, Pathan A, Rab A, Sadiq A, Akram C, Burki I, Shah K. Prevalence of primary multidrug resistance to antituberculosis drugs in Pakistan. Int J Tuberc Lung Dis. 2008;12(3):326-31. 
20. Ullah I, Javaid A, Tahir Z, Ullah O, Shah AA, Hasan F, Ayub N. Pattern of Drug Resistance and Risk Factors Associated with Development of Drug Resistant Mycobacterium tuberculosis in Pakistan. PLoS One. 2016;11(1):e0147529. 